\title{
Laplace Transform Collocation Method for Solving Hyperbolic Telegraph Equation
}

\author{
Adebayo O. Adewumi, Saheed O. Akindeinde, \\ Adebayo A. Aderogba, and Babatunde S. Ogundare
}

Research Group in Computational Mathematics (RGCM), Department of Mathematics, Obafemi Awolowo University, Ile-Ife 220005, Nigeria

Correspondence should be addressed to Babatunde S. Ogundare; bogunda@oauife.edu.ng

Received 13 July 2016; Revised 11 February 2017; Accepted 28 February 2017; Published 10 April 2017

Academic Editor: Bhabani S. Dandapat

Copyright (C) 2017 Adebayo O. Adewumi et al. This is an open access article distributed under the Creative Commons Attribution License, which permits unrestricted use, distribution, and reproduction in any medium, provided the original work is properly cited.

\begin{abstract}
This article presents a new numerical scheme to approximate the solution of one-dimensional telegraph equations. With the use of Laplace transform technique, a new form of trial function from the original equation is obtained. The unknown coefficients in the trial functions are determined using collocation method. The efficiency of the new scheme is demonstrated with examples and the approximations are in excellent agreement with the analytical solutions. This method produced better approximations than the ones produced with the standard weighted residual methods.
\end{abstract}

\section{Introduction}

In this paper, we consider the second-order one-dimensional telegraph equation

$$
\frac{\partial^{2} u}{\partial t^{2}}+\alpha \frac{\partial u}{\partial t}+\beta u=\frac{\partial^{2} u}{\partial x^{2}}+f(x, t),
$$

where $\alpha, \beta$ are known constants and $f(x, t)$ is continuous in the displayed arguments.

Equation (1) describes an electrical signal traveling along a transmission cable; this was first derived in the horse and buggy days of the telegraph (from where it derived its name) and it is still useful for describing long distance power lines and cable TV systems [1].

The study of electric signal in a transmission line, dispersive wave propagation, pulsating blood flow in arteries, and random motion of bugs along a hedge is amongst a host of physical and biological phenomena which can be described by (1). For expository details on the abovementioned phenomena, readers are advised to see [1-4].

Recently, telegraph equation is found to be more suitable than ordinary diffusion equation in modelling reaction diffusion for such branches of science [5]. Without any doubt, (1) and its solution are of great importance in many areas of application. Various analytical and numerical methods have been developed and employed to solve this equation. These include the Method of Weighted Residuals [6], Laplace transform inversion technique with homotopy perturbation method [7], radial basis function method [8], Chebyshev tau method [9], Legendre multiwavelet Galerkin method [10], reciprocity boundary integral equation method [3], Adomian decomposition method [11], unconditionally stable difference scheme [12], and the Reduced Differential Transform Method (RDTM) [13] to mention just a few. Other researchers have also proposed different numerical schemes for solving telegraph equation; for example, Dehghan and Lakestani [14] proposed a method based on Chebyshev cardinal functions to solve one-dimensional hyperbolic telegraph equation, and Javidi [15] used Chebyshev spectral collocation method for computing numerical solution of telegraph equation. Borhanifar and Abazari [16] developed an unconditionally stable parallel difference scheme for telegraph equation. Lakestani and Saray [8] developed a numerical technique for the solution of second-order one-dimensional linear hyperbolic equation. The method consists of expanding the required approximate solution as the elements of interpolating scaling function. In their technique, by using operational 
matrix of derivatives, they reduced the problem to a set of algebraic equations [7]. Hesameddini and Asadolahifard [17] applied the Sinc-Collocation Method to approximate the solution of (1). Mittal and Bhatia [18] and Rashidinia et al. [19] employed the Cubic B-spline Collocation Method (CuBSCM) to approximate the solution of (1). In [20], the authors employed the Fibonacci Polynomials approach to approximate solution of telegraph equations.

Motivated by the works of Odejide and Binuyo [6] where the weighted residual method was applied to the onedimensional telegraph equation, in this work, a new and efficient collocation method based on the Laplace transform is proposed to approximate the solution of (1). This new method shall be called Laplace Transform Collocation Method (LTCM).

The rest of this paper is organized as follows: In Section 2, brief description of the method is presented, and Section 3 is devoted to the error analysis of the method. Implementation of the method using numerical examples is presented in Section 4 while the last section presents our conclusion.

\section{Laplace Transform Collocation Method (LTCM)}

To put emphasis on the essential mathematical details of the new method, we consider the following one-dimensional hyperbolic telegraph equation:

$$
\begin{aligned}
\frac{\partial^{2} u}{\partial t^{2}}+\alpha \frac{\partial u}{\partial t}+\beta u=\frac{\partial^{2} u}{\partial x^{2}} & +f(x, t) \\
x & \in \Omega=[a, b] \subset \Re, 0<t \leq T
\end{aligned}
$$

with the initial conditions

$$
\begin{gathered}
u(x, 0)=g_{1}(x), \quad x \in \Omega \\
u_{t}(x, 0)=g_{2}(x), \quad x \in \Omega
\end{gathered}
$$

and Dirichlet boundary condition

$$
u(x, t)=h(x, t), \quad x \in \partial \Omega, 0<t \leq T,
$$

where $\alpha$ and $\beta$ are known constant coefficients, $f, g_{1}, g_{2}$, and $h$ are known continuous functions in their respective domains, and the function $u$ is unknown.

Taking the Laplace transform of (2), we have

$$
\begin{aligned}
s^{2} U & (x, s)-s u(x, 0)-u_{t}(x, 0) \\
& =\mathscr{L}\left[\frac{\partial^{2} u}{\partial x^{2}}\right]+\mathscr{L}[f(x, t)]-\alpha \mathscr{L}\left[\frac{\partial u}{\partial t}\right]-\beta \mathscr{L}[u] .
\end{aligned}
$$

After simple algebraic simplification, we get

$$
\begin{gathered}
U(x, s)=\frac{1}{s^{2}}\left[s u(x, 0)+u_{t}(x, 0)+\mathscr{L}\left[\frac{\partial^{2} u}{\partial x^{2}}\right]\right. \\
\left.+\mathscr{L}[f(x, t)]-\alpha \mathscr{L}\left[\frac{\partial u}{\partial t}\right]-\beta \mathscr{L}[u]\right] .
\end{gathered}
$$

The function $u(x, t)$ and its derivatives in (6) are thereafter replaced with a trial function of the form

$$
u=u_{0}+\sum_{i=1}^{n} c_{i} u_{i},
$$

where $c_{i}$ are constants to be determined which satisfy the given conditions (3) and (4). Thus, we have the following:

$$
\begin{aligned}
& U(x, s)=\frac{1}{s^{2}}\left[s\left(u_{0}(x, 0)+\sum_{i=1}^{n} c_{i} u_{i}(x, 0)\right)\right. \\
&+\left.\frac{\partial}{\partial t}\left(u_{0}(x, t)+\sum_{i=0}^{n} c_{i} u_{i}(x, t)\right)\right|_{t=0} \\
&+ \mathscr{L}\left[\frac{\partial^{2}}{\partial x^{2}}\left(u_{0}(x, t)+\sum_{i=1}^{n} c_{i} u_{i}(x, t)\right)\right] \\
&+ \mathscr{L}[f(x, t)] \\
&-\alpha \mathscr{L}\left[\frac{\partial}{\partial t}\left(u_{0}(x, t)+\sum_{i=1}^{n} c_{i} u_{i}(x, t)\right)\right] \\
&-\beta \mathscr{L}\left[\frac{\partial}{\partial t}\left(u_{0}(x, t)+\sum_{i=1}^{n} c_{i} u_{i}(x, t)\right)\right] .
\end{aligned}
$$

Taking the inverse Laplace transform of (8), we have

$$
\begin{aligned}
& u_{\text {new }}(x, t)=\mathscr{L}^{-1}\left[\frac{1}{s^{2}}\left(s\left(u_{0}(x, 0)+\sum_{i=1}^{n} c_{i} u_{i}(x, 0)\right)\right)\right. \\
& +\left.\frac{\partial}{\partial t}\left(u_{0}(x, t)+\sum_{i=0}^{n} c_{i} u_{i}(x, t)\right)\right|_{t=0} \\
& +\mathscr{L}\left[\frac{\partial^{2}}{\partial x^{2}}\left(u_{0}(x, t)+\sum_{i=1}^{n} c_{i} u_{i}(x, t)\right)\right] \\
& +\mathscr{L}[f(x, t)] \\
& -\alpha \mathscr{L}\left[\frac{\partial}{\partial t}\left(u_{0}(x, t)+\sum_{i=1}^{n} c_{i} u_{i}(x, t)\right)\right] \\
& \left.-\beta \mathscr{L}\left[\frac{\partial}{\partial t}\left(u_{0}(x, t)+\sum_{i=1}^{n} c_{i} u_{i}(x, t)\right)\right]\right]
\end{aligned}
$$

Substituting (9) into (2), we get

$$
\begin{gathered}
\frac{\partial^{2}}{\partial t^{2}} u_{\text {new }}(x, t)+\alpha \frac{\partial}{\partial t} u_{\text {new }}(x, t)+\beta u_{\text {new }}(x, t) \\
=\frac{\partial^{2}}{\partial x^{2}} u_{\text {new }}(x, t)+f(x, t) .
\end{gathered}
$$

Now, collocating (10) at points $x=x_{j}$, we have

$$
\begin{aligned}
& \frac{\partial^{2}}{\partial t^{2}} u_{\text {new }}\left(x_{j}, t\right)+\alpha \frac{\partial}{\partial t} u_{\text {new }}\left(x_{j}, t\right)+\beta u_{\text {new }}\left(x_{j}, t\right) \\
& \quad=\frac{\partial^{2}}{\partial x_{j}^{2}} u_{\text {new }}\left(x_{j}, t\right)+f\left(x_{j}, t\right)
\end{aligned}
$$


where

$$
x_{j}=a+\frac{(b-a)}{n+1} j, \quad j=1,2, \ldots, n .
$$

Thus, (11) constitutes $n$-equations in $n$-unknowns which can be determined by using Gaussian elimination method. Substituting these coefficients into (9) gives the approximate solutions.

\section{Error Analysis of Laplace Transform Collocation Method (LTCM)}

Let us define the error function $e_{n}(x, t)=u(x, t)-u_{n}(x, t)$, where $u(x, t)$ and $u_{n}(x, t)$ denote, respectively, the exact and approximate solution obtained via our proposed method. In line with [20], we define the residual function

$$
R_{n}(x, t)=L\left[u_{n}(x, t)\right]-f(x, t),
$$

where

$$
L\left[u_{n}(x, t)\right]=\frac{\partial^{2} u_{n}}{\partial t^{2}}+\alpha \frac{\partial u_{n}}{\partial t}+\beta u_{n}-\frac{\partial^{2} u_{n}}{\partial x^{2}}
$$

It then follows that

$$
\frac{\partial^{2} u_{n}}{\partial t^{2}}+\alpha \frac{\partial u_{n}}{\partial t}+\beta u_{n}-\frac{\partial^{2} u_{n}}{\partial x^{2}}=f+R_{n}
$$

subject to initial conditions

$$
\begin{aligned}
u_{n}(x, 0) & =g_{1}(x) \\
\left(u_{n}\right)_{t}(x, 0) & =g_{2}(x), \\
u_{n}(x, t) & =h(x), \quad x \in \partial \Omega .
\end{aligned}
$$

Now since $L$ is a linear operator, we obtain for the error function $e_{n}(x, t)$

$$
\begin{aligned}
& \frac{\partial^{2} e_{n}(x, t)}{\partial t^{2}}+\alpha \frac{\partial e_{n}(x, t)}{\partial t}+\beta e_{n}(x, t)-\frac{\partial^{2} e_{n}(x, t)}{\partial x^{2}} \\
& \quad=-R_{n}(x, t)
\end{aligned}
$$

with the homogeneous conditions

$$
\begin{aligned}
e_{n}(x, 0) & =0, \\
\left(e_{n}\right)_{t}(x, 0) & =0, \\
e_{n}(a, t) & =0, \\
e_{n}(b, t) & =0 .
\end{aligned}
$$

By solving (17) subject to the homogeneous conditions above, we obtain the error function $e_{n}(x, t)$. This allows us to compute $u(x, t)=u_{n}(x, t)+e_{n}(x, t)$ even for problems without known exact solutions.

\section{Numerical Examples}

In this section, we implement the new method on some examples to test its efficiency and applicability.

Example 1. We consider the case in which $\alpha=4, \beta=2$, and $f(x, t)=0$, and (2) becomes (see [6])

$$
\frac{\partial^{2} u}{\partial t^{2}}+4 \frac{\partial u}{\partial t}+2 u=\frac{\partial^{2} u}{\partial x^{2}}
$$

subject to

$$
\begin{aligned}
& u(x, 0)=\sin (x), \quad 0 \leq x \leq \pi \\
& u_{t}(x, 0)=-\sin (x), \quad 0 \leq x \leq \pi, \\
& u(0, t)=0, \\
& u(\pi, t)=0,
\end{aligned}
$$

$$
t>0 \text {. }
$$

The exact solution is given by $u(x, t)=e^{-t} \sin (x)$.

We assume the trial function of the form:

$$
\begin{aligned}
u(x, t)= & (1-t) \sin (x)+c_{1} x^{2}(x-\pi) t^{2} \\
& +c_{2} x(x-\pi)^{2} t^{2} .
\end{aligned}
$$

Taking the Laplace transform of (19), we get

$$
\begin{aligned}
& s^{2} U(x, s)-s u(x, 0)-u_{t}(x, 0)+4 \mathscr{L}\left[\frac{\partial}{\partial t} u(x, t)\right] \\
& +2 \mathscr{L}[u(x, t)]=\mathscr{L}\left[\frac{\partial^{2}}{\partial x^{2}} u(x, t)\right] .
\end{aligned}
$$

Rearranging (22), we have

$$
\begin{aligned}
& U(x, s)=\frac{1}{s^{2}}[s \sin (x)-\sin (x) \\
& -4 \mathscr{L}\left[2 c_{1} x^{2}(x-\pi) t+2 c_{2} x(x-\pi)^{2} t-\sin (x)\right] \\
& \quad-2 \mathscr{L}\left[c_{1} x^{2}(x-\pi) t^{2}+c_{2} x(x-\pi)^{2} t^{2}+(1-t)\right. \\
& \quad \cdot \sin (x)]+\mathscr{L}\left[2 c_{1}(x-\pi) t^{2}+4 c_{1} x t^{2}\right. \\
& \left.\left.+4 c_{2}(x-\pi) t^{2}+2 c_{2} x t^{2}-(1-t) \sin (x)\right]\right] .
\end{aligned}
$$

Taking the inverse Laplace transform of (23), we have the following new trial solution:

$$
\begin{aligned}
u_{\text {new }}( & x, t) \\
= & \left(-\frac{4}{3} t^{2} c_{1}-\frac{1}{6} t^{4} c_{1}-\frac{4}{3} t^{3} c_{2}-\frac{1}{6} t^{4} c_{2}\right) x^{3} \\
& +\left(\frac{8}{3} t^{3} c_{2} \pi+\frac{4}{3} t^{3} c_{1} \pi+\frac{1}{6} t^{4} c_{1} \pi+\frac{1}{3} t^{4} c_{2} \pi\right) x^{2} \\
& +\left(\frac{1}{2} t^{4} c_{2}-\frac{1}{6} t^{4} c_{2} \pi^{2}+\frac{1}{2} t^{4} c_{1}-\frac{4}{3} t^{3} c_{2} \pi^{2}\right) x
\end{aligned}
$$




$$
\begin{aligned}
& +\left(1+\frac{1}{2} t^{3}+\frac{1}{2} t^{2}-t\right) \sin (x) \\
& -\left(\frac{1}{3} c_{2}+\frac{1}{6} c_{1}\right) t^{4} \pi .
\end{aligned}
$$

Substituting (24) into (19), we have the following residual function:

$$
\begin{aligned}
& R\left(x, t, c_{1}, c_{2}\right)=\left(-\frac{16}{3} t^{2} c_{1}-\frac{1}{3} t^{4} c_{1}-\frac{16}{3} t^{3} c_{2}-\frac{1}{3} t^{4} c_{2}\right. \\
& \left.-18 c_{2} t^{2}-18 c_{1} t^{2}-8 c_{1} t-8 c_{2} t\right) x^{3}+\left(16 c_{2} t \pi\right. \\
& +\frac{2}{3} t^{4} c_{2} \pi+8 c_{1} t \pi+18 t^{2} c_{1} \pi+\frac{1}{3} t^{4} c_{1} \pi+\frac{16}{3} t^{3} c_{1} \pi \\
& \left.+36 t^{2} c_{2} \pi+\frac{32}{3} t^{3} c_{2} \pi\right) x^{2}+\left(6 c_{2} t^{2}+2 t^{4} c_{2}\right. \\
& -18 t^{2} c_{2} \pi^{2}+6 c_{1} t^{2}-8 c_{2} t \pi^{2}-\frac{16}{3} t^{3} c_{2} \pi^{2}+2 t^{4} c_{2} \\
& \left.-\frac{1}{3} t^{4} c_{2} \pi^{2}+16 t^{3} c_{1}+16 t^{3} c_{2}\right) x-\left(\frac{32}{3} t^{3} c_{2}+2 t^{2} c_{2}\right. \\
& \left.+\frac{4}{3} t^{4} c_{2}+4 t^{2} c_{2}\right) \pi+\left(4 t+\frac{3}{2} t^{3}+\frac{15}{2} t^{2}\right) \sin (x) \\
& -\left(\frac{16}{3} t^{3} \pi+\frac{2}{3} t^{4} \pi\right) c_{1} .
\end{aligned}
$$

Collocating (25) at equally spaced points $x_{1}=\pi / 3$ and $x_{2}=2 \pi / 3$ for $t=0.01$ and equating to zero, we then use the Gaussian elimination method to solve the two systems of equations and obtained $c_{1}=-0.062540123863941896592$ and $c_{2}=0.062540123863941870829$.

Substituting these values into (24), we obtain the following approximate solution:

$$
\begin{aligned}
& u(x, t)=\left(3.4351 \times 10^{-17} t^{3}+4.294 \times 10^{-18} t^{4}\right) x^{3} \\
& +\left(0.083386831818589126754 t^{3} \pi\right. \\
& \left.+0.010423353977323640844 t^{4} \pi\right) x^{2}+(-1.2882 \\
& \quad \times 10^{-17} t^{4}-0.010423353977323645138 t^{4} \pi^{2} \\
& \left.-0.083386831818589161105 t^{3} \pi^{2}\right) x+\left(1+\frac{1}{2} t^{3}\right. \\
& \left.+\frac{1}{2} t^{2}-t\right) \sin (x) \\
& \quad-0.010423353977323640844 t^{4} \pi
\end{aligned}
$$

Table 1 gives the comparison between the new method (LTCM) and the Method of Weighted Residual (MWR) for Example 1. Figure 1 shows the comparison of approximate and exact solution for Example 1. The error plot for Example 1 is shown in Figure 2.

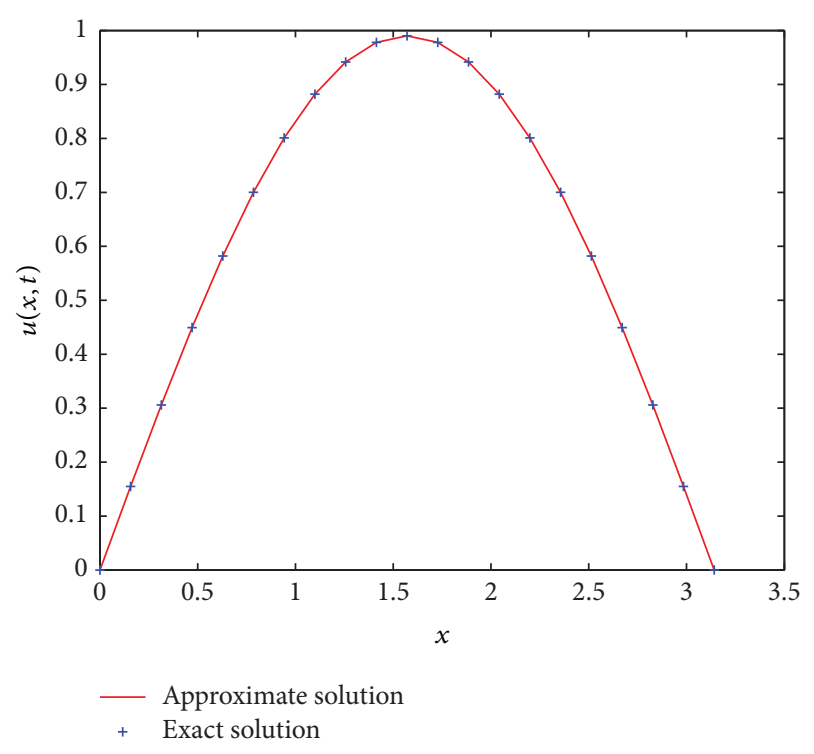

FIGURE 1: Comparison of approximate and exact solution for Example 1.

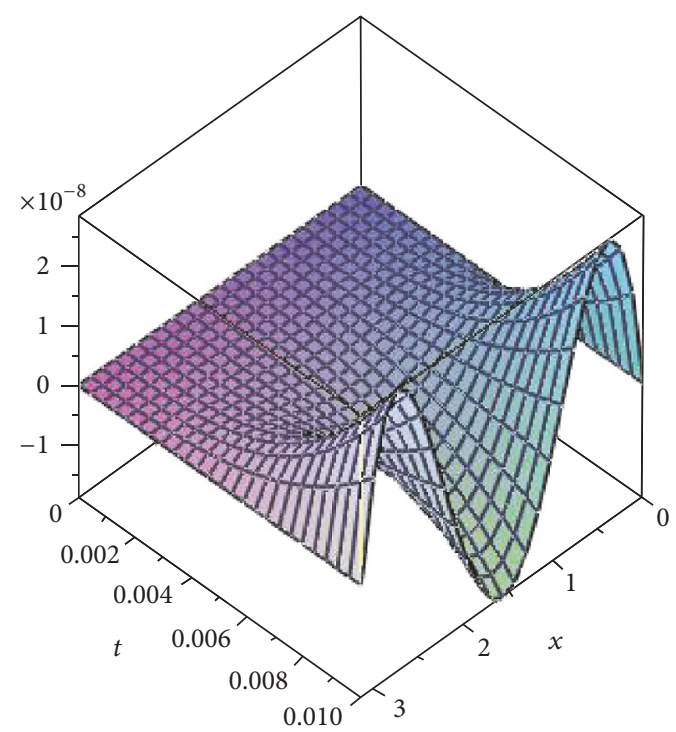

Figure 2: The error plot for Example 1.

Example 2. We consider next the case in which $\alpha=6, \beta=2$, and $f(x, t)=2 e^{-t} \sin (x)$, and (2) becomes

$$
\frac{\partial^{2} u}{\partial t^{2}}+6 \frac{\partial u}{\partial t}+2 u=\frac{\partial^{2} u}{\partial x^{2}}-2 e^{-t} \sin (x)
$$

subject to

$$
\begin{aligned}
u(x, 0) & =\sin (x), \quad 0 \leq x \leq \pi \\
u_{t}(x, 0) & =-\sin (x), \quad 0 \leq x \leq \pi, \\
u(0, t) & =0, \\
u(\pi, t) & =0,
\end{aligned}
$$

$$
t>0 \text {. }
$$

The exact solution is given by $u_{e}=e^{-t} \sin (x)$ [6]. 
TABLE 1: Comparison of LTCM with MWR at $t=0.01$ for Example 1.

\begin{tabular}{llllr}
\hline$x$ & Exact solution & LTCM solution & Error of LTCM & Error in [6] \\
\hline 0.0 & 0.000000000000 & $3.27459 \times 10^{-10}$ & $3.2746 E-10$ & 0.0000 \\
$\frac{11}{14}$ & 0.700292224223 & 0.700292209635 & $1.4588 E-08$ & $9.3980 E-06$ \\
$\frac{11}{7}$ & 0.990049635871 & 0.990049654607 & $1.8737 E-08$ & $1.5949 E-06$ \\
$\frac{33}{14}$ & 0.699406712281 & 0.699406697618 & $1.4663 E-08$ & $9.4560 E-07$ \\
$\frac{22}{7}$ & -0.001251907055 & -0.001251907182 & $1.2754 E-10$ & $1.4682 E-08$ \\
\hline
\end{tabular}

We assume an approximate solution of the form

$$
\begin{aligned}
u(x, t)= & (1-t) \sin (x)+c_{1} x^{2}(x-\pi) t^{2} \\
& +c_{2} x(x-\pi)^{2} t^{2}
\end{aligned}
$$

Following the same procedure as discussed in Example 1, we have the following new trial function:

$$
\begin{aligned}
u_{\text {new }}= & \left(-2 t^{3} c_{1}-\frac{1}{6} t^{4} c_{1}+2 t^{3} c_{2}-\frac{1}{6} t^{4} c_{2}\right) x^{3} \\
& +\left(4 t^{3} c_{2} \pi+2 t^{3} c_{1} \pi+\frac{1}{6} t^{4} c_{1} \pi+\frac{1}{3} t^{4} c_{2} \pi\right) x^{2} \\
& +\left(\frac{1}{2} t^{4} c_{2}-\frac{1}{6} t^{4} c_{2} \pi+\frac{1}{2} t^{4} c_{1}-2 t^{3} c_{2} \pi^{2}\right) x \\
& +\left(3-2 e^{-t}+\frac{1}{2} t^{3}+\frac{3}{2} t^{2}-3 t\right) \sin (x) \\
& -\left(\frac{1}{3} t^{4} c_{2}+\frac{1}{6} t^{4} c_{1}\right) \pi
\end{aligned}
$$

Substituting (30) into (27) and collocating at points $x_{1}=\pi / 3$ and $x_{2}=2 \pi / 3$ for $t=0.01$ and also equating to zero and solving the resulting equations by Gaussian elimination method, we obtain $c_{1}=-0.062538703934436983406$ and $c_{2}=0.062538703934436957732$.

Similarly, substituting these values into (30), we determine the following approximate solution:

$$
\begin{aligned}
& u(x, t)=\left(5.135 \times 10^{-17} t^{3}+4.279 \times 10^{-18} t^{4}\right) x^{3} \\
& +\left(0.12507740786887386412 t^{3} \pi\right. \\
& +0.0010423117322406159622 t^{4} \pi^{2} \\
& \left.\quad-0.12507740786887391546 t^{3} \pi^{2}\right) x+\left(3-2 e^{-t}\right. \\
& \left.+\frac{1}{2} t^{3}+\frac{3}{2} t^{2}-3 t\right) \sin (x) \\
& \quad-0.010423117322406155343 t^{4} \pi .
\end{aligned}
$$

Comparison between the approximation with the new method (LTCM) and the conventional Method of Weighted Residual (MWR) for Example 2 is given in Table 2. Figure 3

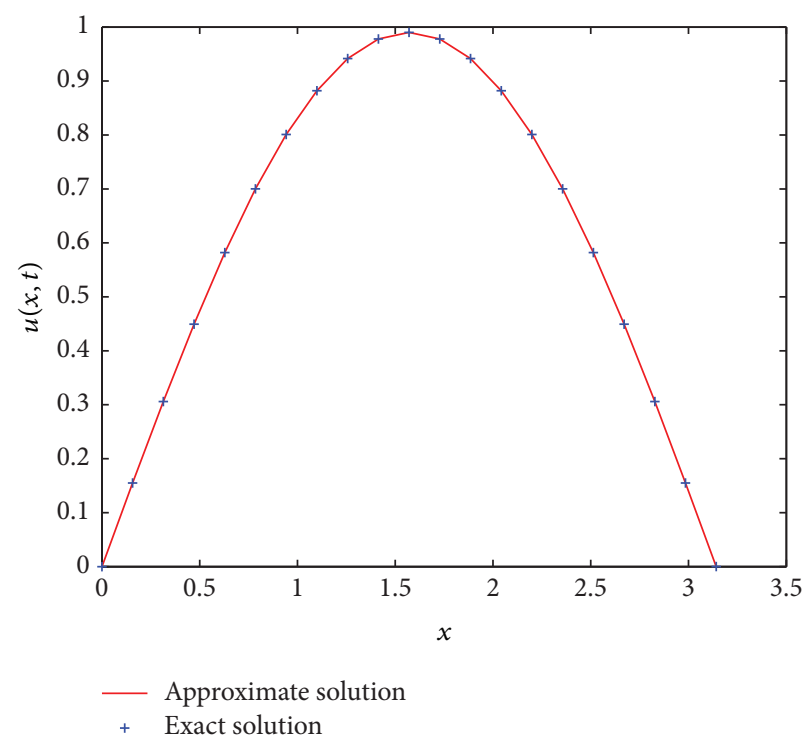

FIGURE 3: Comparison of approximate and exact solution for Example 2.

shows the comparison of approximate and exact solution for Example 2. The error plot for Example 2 is shown in Figure 4.

Example 3. We now consider the case in which $\alpha=1, \beta=1$, and $f(x, t)=\left(2-2 t+t^{2}\right)\left(x-x^{2}\right) e^{-t}$, and we solve

$$
\begin{aligned}
\frac{\partial^{2} u}{\partial t^{2}}+\frac{\partial u}{\partial t}+u= & \frac{\partial^{2} u}{\partial x^{2}}+\left(2-2 t+t^{2}\right)\left(x-x^{2}\right) e^{-t} \\
& +2 t e^{-t}
\end{aligned}
$$

subject to

$$
\begin{aligned}
& u(x, 0)=0, \quad 0 \leq x \leq 1, \\
& u_{t}(x, 0)=0, \quad 0 \leq x \leq 1, \\
& u(0, t)=0 \\
& u(1, t)=0
\end{aligned}
$$

$$
t>0 \text {. }
$$

The exact solution is given by $u_{e}=\left(x-x^{2}\right) t^{2} e^{-t}[6]$. 
TABLE 2: Comparison of LTCM with MWR at $t=0.01$ for Example 2.

\begin{tabular}{lcccc}
\hline$x$ & Exact solution & LTCM solution & Error of LTCM & Error in [6] \\
\hline 0.0 & 0.000000000000 & $3.2745 \times 10^{-10}$ & $3.2745 E-10$ & 0.0000 \\
$\frac{11}{14}$ & 0.700292224223 & 0.700292202383 & $2.1840 E-08$ & $1.3745 E-04$ \\
$\frac{11}{7}$ & 0.990049635871 & 0.990049663942 & $2.8070 E-08$ & $1.8038 E-04$ \\
$\frac{33}{14}$ & 0.699406712281 & 0.699406690328 & $2.1952 E-08$ & $1.3731 E-04$ \\
$\frac{22}{7}$ & -0.001251907055 & -0.001251907082 & $2.7466 E-11$ & $3.0778 E-07$ \\
\hline
\end{tabular}

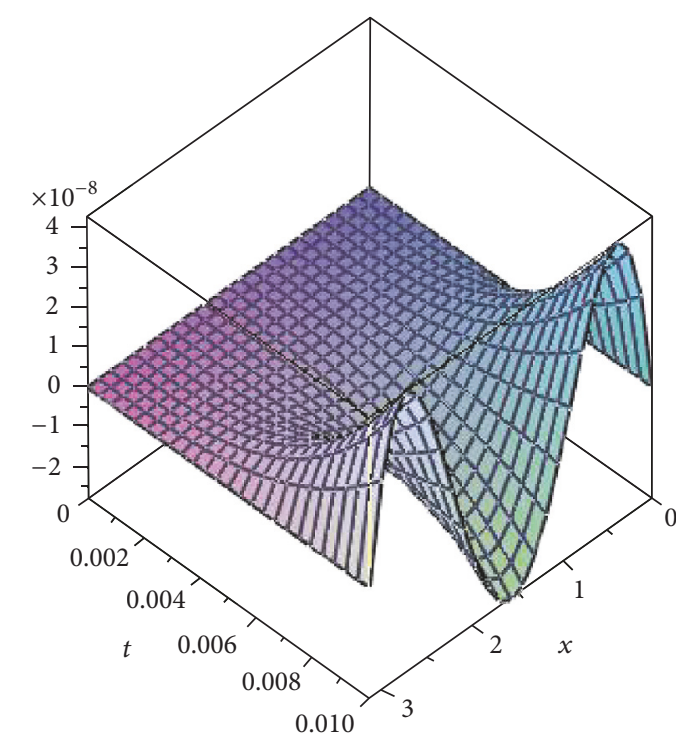

FIgURE 4: The error plot for Example 2.

Similarly, taking

$$
u(x, t)=c_{1} x^{2}(x-1) t^{2}+c_{2} x(x-1)^{2} t^{2},
$$

and following the same procedure as in Example 2, we have the following new trial solution:

$$
\begin{aligned}
& u(x, t)=\left(-\frac{1}{12} t^{4} c_{1}-\frac{1}{3} t^{3} c_{2}-\frac{1}{3} t^{3} c_{1}-\frac{1}{12} t^{4} c_{2}\right) x^{3} \\
& +\left(4+\frac{1}{3} t^{3} c_{1}-2 t-t^{2} e^{-t}+\frac{1}{12} t^{4} c_{1}-4 e^{-t}+\frac{2}{3} t^{3} c_{2}\right. \\
& \left.-2 t e^{-t}+\frac{1}{6} t^{4} c_{2}\right) x^{2}+\left(2 t e^{-t}-\frac{1}{3} t^{3} c_{2}-4+2 t\right. \\
& \left.+t^{2} e^{-t}+\frac{1}{2} t^{4} c_{1}+4 e^{-t}+\frac{5}{12} t^{4} c_{2}\right) x-12-\frac{1}{3} t^{4} c_{2} \\
& -4 t-\frac{1}{6} t^{4} c_{1}+\left(12+2 t^{2}+8 t\right) e^{-t}
\end{aligned}
$$

Substituting (35) into (32) and collocating at points $x_{1}=1 / 3$ and $x_{2}=2 / 3$ and later solving the resulting equations, we obtain the following values for $c_{1}$ and $c_{2}: c_{1}=-0.98535992634058334369$ and $c_{2}=$ 0.98535992634058334369 .

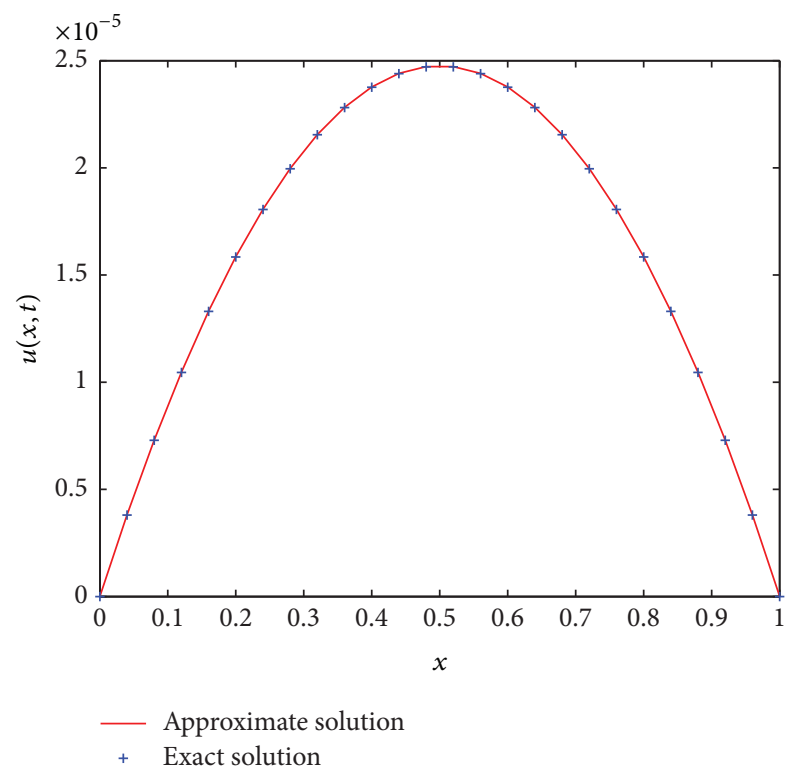

FIgURE 5: Comparison of approximate and exact solution for Example 3.

Substituting these values into (35), we have

$$
\begin{aligned}
& u(x, t)=\left(4+0.32845330878019444789 t^{3}-2 t\right. \\
& -t^{2} e^{-t}+0.082113327195048611976 t^{4}-4 e^{-t} \\
& \left.-2 t e^{-t}\right) x^{2}+\left(2 t e^{-t}-0.32845330878019444790 t^{3}\right. \\
& \quad-4+2 t+t^{2} e^{-t}-0.08211332719504861197 t^{4} \\
& +4 e-t) x+\left(12+2 t^{2}+8 t\right) e^{-t}+4 t \\
& -0.16422665439009722395 t^{4}-12
\end{aligned}
$$

Comparison between the approximation with the new method (LTCM) and the conventional Method of Weighted Residual (MWR) for Example 3 is given in Table 3. Figure 5 shows the comparison of approximate and exact solution for Example 3.

The error plot for Example 3 is shown in Figure 6. 
TABLE 3: Comparison of LTCM with MWR at $t=0.01$ for Example 3.

\begin{tabular}{lcccc}
\hline$x$ & Exact solution & LTCM solution & Error of LTCM & Error in [6] \\
\hline 0.0 & 0.000000000000 & $1.4433 \times 10^{-11}$ & $1.4433 E-11$ & 0.0000 \\
$\frac{1}{4}$ & 0.000018563434 & 0.000018563898 & $4.6391 E-10$ & $3.6741 E-07$ \\
$\frac{1}{2}$ & 0.000024751245 & 0.000024751859 & $6.1374 E-10$ & $4.8988 E-07$ \\
$\frac{3}{4}$ & 0.000018563434 & 0.000018563898 & $4.6391 E-10$ & $3.6741 E-07$ \\
1 & 0.000000000000 & $1.4433 \times 10^{-11}$ & $1.4433 E-11$ & 0.0000 \\
\hline
\end{tabular}

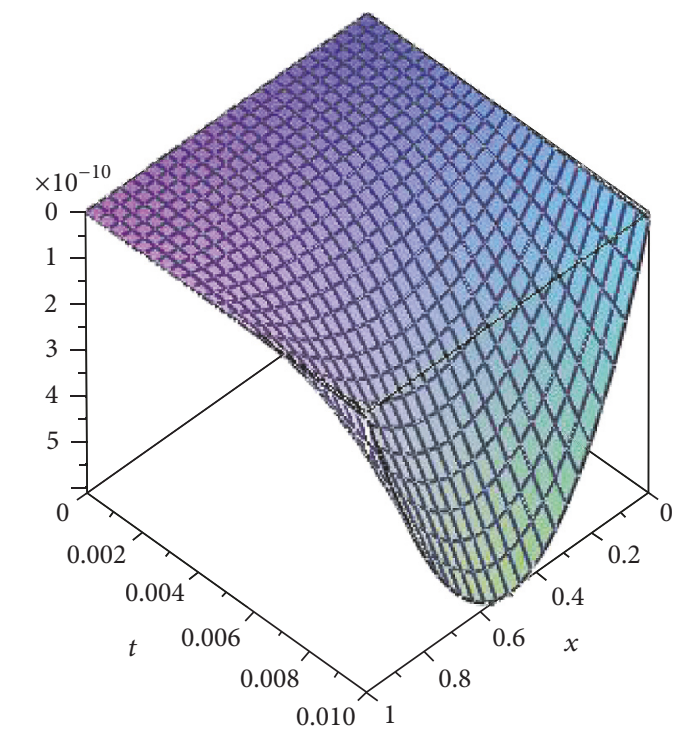

FIgURE 6: The error plot for Example 3.

Example 4. We consider the case in which $\alpha=1, \beta=1$, and $f(x, t)=x^{2}+t-1$, and we have

$$
\frac{\partial^{2} u}{\partial t^{2}}+\frac{\partial u}{\partial t}+u=\frac{\partial^{2} u}{\partial x^{2}}+x^{2}+t-1
$$

subject to

$$
\begin{aligned}
& u(x, 0)=x^{2}, \quad 0 \leq x \leq 1, \\
& u_{t}(x, 0)=1, \quad 0 \leq x \leq 1, \\
& u(0, t)=t \\
& u(1, t)=1+t
\end{aligned}
$$

$$
t>0
$$

The exact solution is given by $u_{e}=x^{2}+t[6]$.

We assume a trial solution of the form:

$$
u(x, t)=x^{2}+t+c_{1} x^{2}(x-1) t^{2}+c_{2} x(x-1)^{2} t^{2}
$$

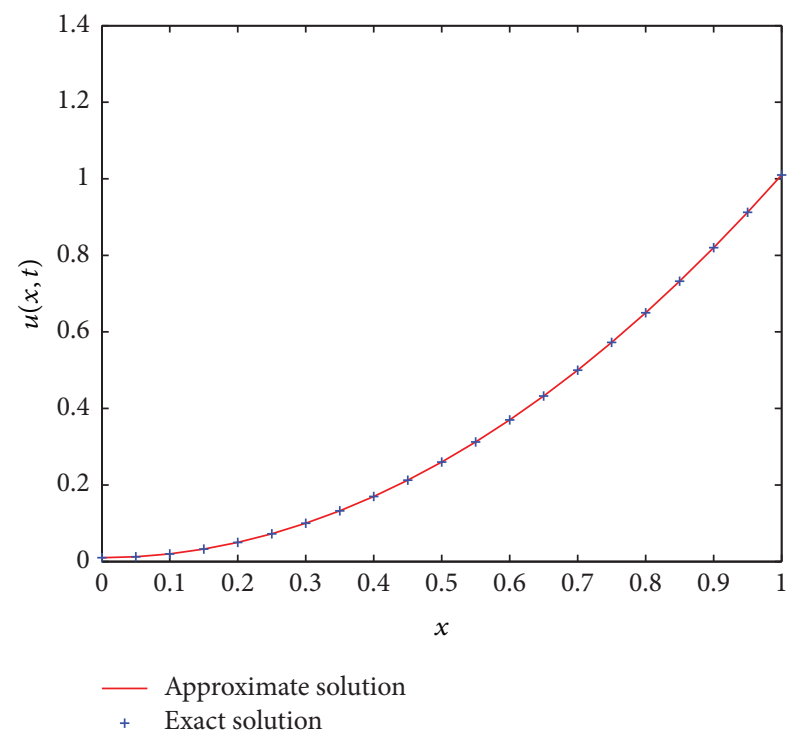

Figure 7: Comparison of approximate and exact solution for Example 4.

Following the same procedure as in other examples, we obtain a new form of trial solution which is given as

$$
\begin{aligned}
u(x, t)= & \left(-\frac{1}{12} c_{1} t^{4}-\frac{1}{3} c_{2} t^{3}-\frac{1}{12} c_{2} t^{4}-\frac{1}{3} c_{1} t^{3}\right) x^{3} \\
& +\left(1+\frac{1}{3} c_{1} t^{3}+\frac{1}{12} c_{1} t^{4}+\frac{2}{3} c_{2} t^{3}+\frac{1}{6} c_{2} t^{4}\right) x^{2} \\
& +\left(\frac{5}{12} c_{2} t^{4}+\frac{1}{2} c_{1} t^{4}-\frac{1}{3} c_{2} t^{3}\right) x+t \\
& -\left(\frac{1}{6} c_{1}-\frac{1}{3} c_{2}\right) t^{4} .
\end{aligned}
$$

Substituting (40) into (37) yields a residual function which is then collocated at points $x_{1}=1 / 3$ and $x_{2}=2 / 3$ for $t=0.01$. Solving the resulting equations, we obtain $c_{1}=c_{2}=0.0000$.

Substituting these values into (40), we have $x^{2}+t$ which is the exact solution.

Table 4 gives the comparison between approximation with the new method (LTCM) and the Method of Weighted Residual (MWR) for Example 4. Figure 7 shows the comparison of approximate and exact solution for Example 4.

The absolute error plot for Example 4 is shown in Figure 8. 
TABLE 4: Comparison of LTCM with MWR at $t=0.01$ for Example 4.

\begin{tabular}{|c|c|c|c|c|}
\hline$x$ & Exact solution & LTCM solution & Error of LTCM & Error in [6] \\
\hline 0.0 & 0.010000000000 & 0.010000000000 & 0.0000 & 0.0000 \\
\hline$\frac{1}{4}$ & 0.072500000000 & 0.072500000000 & 0.0000 & 0.0000 \\
\hline$\frac{1}{2}$ & 0.260000000000 & 0.260000000000 & 0.0000 & 0.0000 \\
\hline$\frac{3}{4}$ & 0.572500000000 & 0.572500000000 & 0.0000 & 0.0000 \\
\hline 1 & 1.010000000000 & 1.010000000000 & 0.0000 & 0.0000 \\
\hline
\end{tabular}

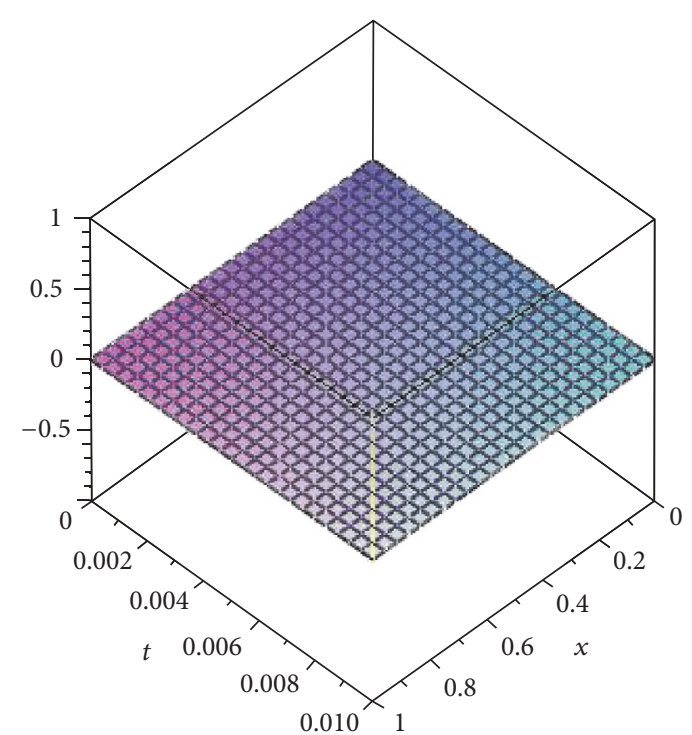

FIgURE 8: The error plot for Example 4.

\section{Conclusion}

In this paper, we adopted a combination of Laplace transform scheme and collocation method to develop a new numerical method for solving one-dimensional linear hyperbolic telegraph equation. Four numerical examples were considered to demonstrate the efficiency and accuracy of the method. It is observed from the solution process that the present method provides a good approximate solution in comparison to the exact solution as it can be seen in Figures 1, 3, 5, and 7 with the absolute error plots displayed in Figures 2, 4,6 , and 8, respectively. Our approximations are compared with the results obtained by Odejide and Binuyo [6] and from the tables of results (Tables 1-4) it is evident that the LTCM produced better results than the MWR. The method proposed in this paper will be extended to solve parabolic Volterra integrodifferential equation in future work.

\section{Conflicts of Interest}

The authors declare that there are no conflicts of interest regarding the publication of this paper.

\section{References}

[1] H. Pascal, "Pressure wave propagation in a fluid flowing through a porous medium and problems related to interpretation of Stoneley's wave attenuation in acoustical well logging," International Journal of Engineering Science, vol. 24, no. 9, pp. 15531570, 1986.

[2] G. Bohme, Non-Newtonian Fluid Mechanics, vol. 31 of NorthHolland Series in Applied Mathematics and Mechanics, NorthHolland, Amsterdam, The Netherlands, 1987.

[3] M. Dehghan and A. Ghesmati, "Solution of the second-order one-dimensional hyperbolic telegraph equation by using the dual reciprocity boundary integral equation (DRBIE) method," Engineering Analysis with Boundary Elements, vol. 34, no. 1, pp. 51-59, 2010.

[4] R. K. Mohanty and M. K. Jain, "An unconditionally stable alternating direction implicit scheme for the two space dimensional linear hyperbolic equation," Numerical Methods for Partial Differential Equations, vol. 17, no. 6, pp. 684-688, 2001.

[5] M. Dehghan and A. Shokri, "A numerical method for solving the hyperbolic telegraph equation," Numerical Methods for Partial Differential Equations, vol. 24, no. 4, pp. 1080-1093, 2008.

[6] S. A. Odejide and A. O. Binuyo, "Numerical solution of hyperbolic telegraph equation using method of weighted residuals," International Journal of Nonlinear Science, vol. 18, no. 1, pp. 6570, 2014.

[7] M. Javidi and N. Nyamoradi, "Numerical solution of telegraph equation by using LT inversion technique," International Journal of Advanced Mathematical Sciences, vol. 1, no. 2, pp. 64-77, 2013.

[8] M. Lakestani and B. N. Saray, "Numerical solution of telegraph equation using interpolating scaling functions," Computers \& Mathematics with Applications, vol. 60, no. 7, pp. 1964-1972, 2010.

[9] A. Saadatmandi and M. Dehghan, "Numerical solution of hyperbolic telegraph equation using the Chebyshev Tau method," Numerical Methods for Partial Differential Equations, vol. 26, no. 1, pp. 239-252, 2010.

[10] S. A. Yousefi, "Legendre multiwavelet Galerkin method for solving the hyperbolic telegraph equation," Numerical Methods for Partial Differential Equations, vol. 26, no. 3, pp. 535-543, 2010.

[11] M. A. Abdou, "Adomian decomposition method for solving the telegraph equation in charged particle transport," Journal of Quantitative Spectroscopy and Radiative Transfer, vol. 95, no. 3, pp. 407-414, 2005.

[12] F. Gao and C. Chi, "Unconditionally stable difference schemes for a one-space-dimensional linear hyperbolic equation," 
Applied Mathematics and Computation, vol. 187, no. 2, pp. 12721276, 2007.

[13] V. K. Srivastava, M. K. Awasthi, R. K. Chaurasia, and M. Tamsir, "The telegraph equation and its solution by reduced differential transform method," Modelling and Simulation in Engineering, vol. 2013, Article ID 746351, 6 pages, 2013.

[14] M. Dehghan and M. Lakestani, “The use of Chebyshev cardinal functions for solution of the second-order one-dimensional telegraph equation," Numerical Methods for Partial Differential Equations, vol. 25, no. 4, pp. 931-938, 2009.

[15] M. Javidi, "Chebyshev spectral collocation method for computing numerical solution of telegraph equation," Computational Methods for Differential Equations, vol. 1, no. 1, pp. 16-29, 2013.

[16] A. Borhanifar and R. Abazari, "An unconditionally stable parallel difference scheme for telegraph equation," Mathematical Problems in Engineering, vol. 2009, Article ID 969610, 17 pages, 2009.

[17] E. Hesameddini and E. Asadolahifard, "The sinc-collocation method for solving the telegraph equation," Journal of Computer Engineering and Informatics, vol. 1, no. 1, pp. 13-17, 2013.

[18] R. C. Mittal and R. Bhatia, "Numerical Solution of second order hyperbolic telegraph equation via new cubic trigonometric Bsplines approach," Applied Mathematics and Computation, vol. 220, no. 1, pp. 496-506, 2013.

[19] J. Rashidinia, S. Jamalzadeh, and F. Esfahani, "Numerical solution of one-dimensional telegraph equation using cubic B-spline collocation method," Journal of Interpolation and Approximation in Scientific Computing, vol. 2014, Article ID jiasc-00042, 8 pages, 2014.

[20] A. K. Bahşı and S. Yalçınbaş, "A new algorithm for the numerical solution of telegraph equations by using fibonacci polynomials," Mathematical and Computational Applications, vol. 21, no. 2, p. 15, 2016. 


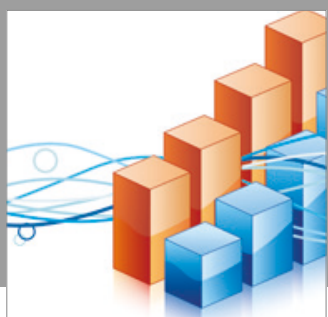

Advances in

Operations Research

vatersals

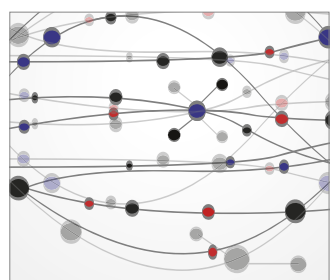

\section{The Scientific} World Journal
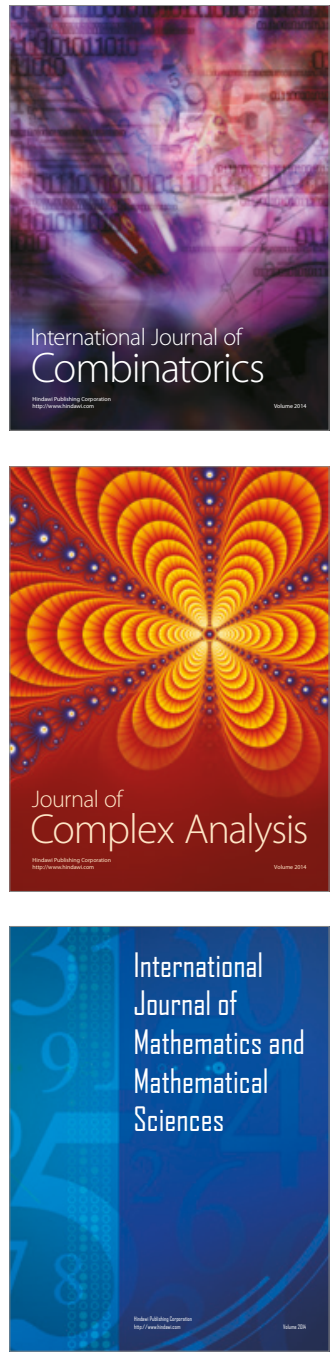
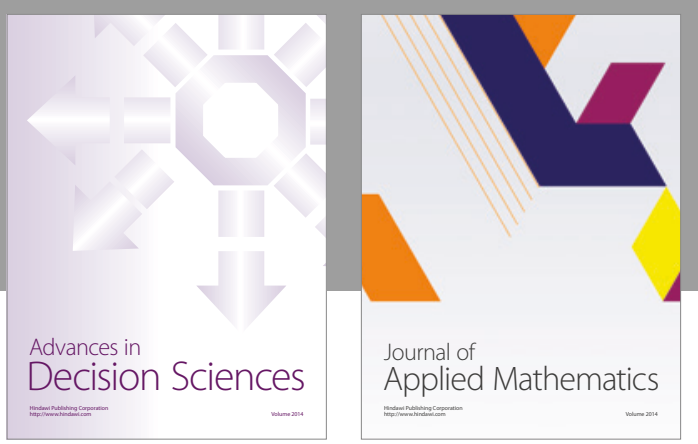

Algebra

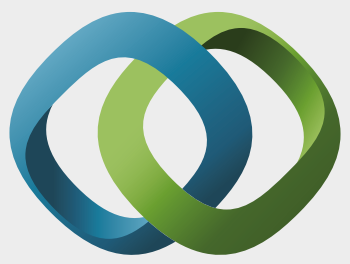

\section{Hindawi}

Submit your manuscripts at

https://www.hindawi.com
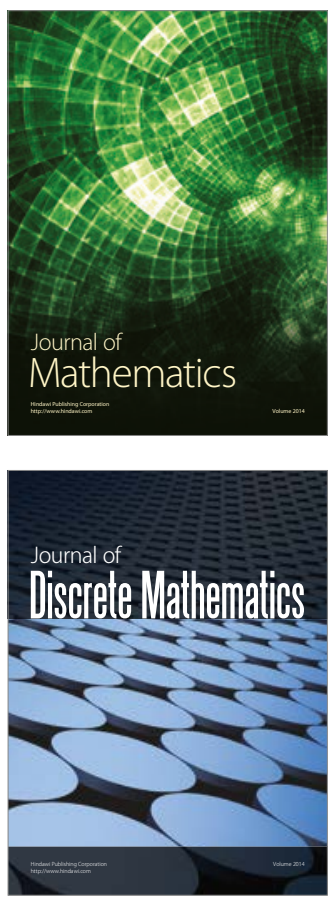

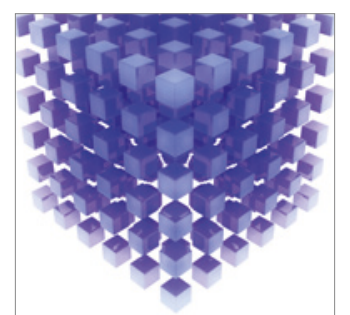

Mathematical Problems in Engineering
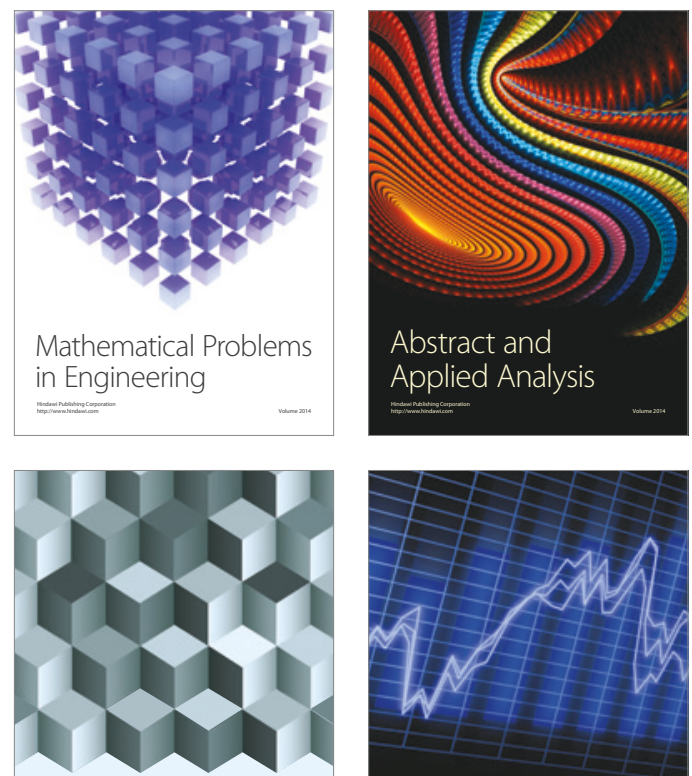

Journal of

Function Spaces

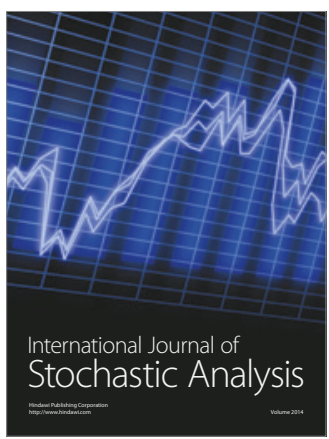

Probability and Statistics
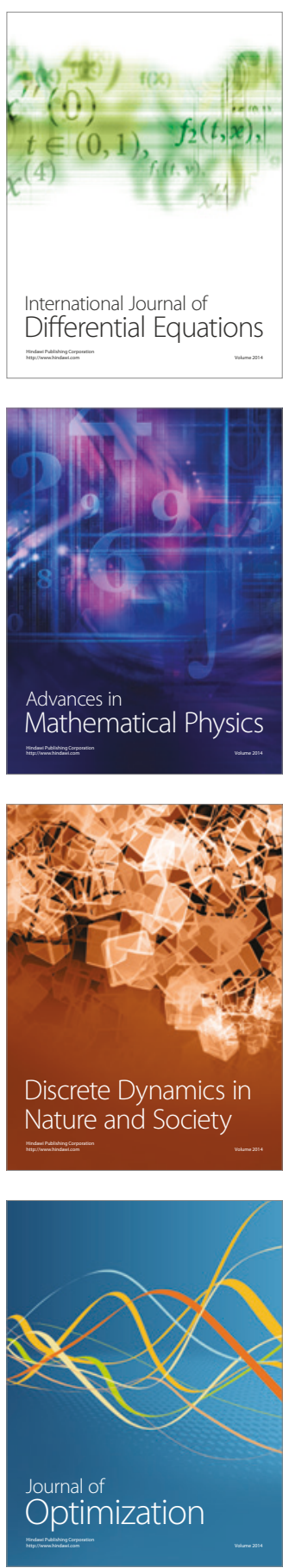